\title{
LOW COST INTEGRATED NAVIGATION SYSTEM FOR UNMANNED VESSEL
}

\author{
Changsong Yang ${ }^{1 *}$ \\ Qi Wang ${ }^{2}$ \\ ${ }^{1}$ School of Information \& Control, Nanjing University of Information Science \& Technology, China \\ ${ }^{2}$ School of Computer \& Software, Nanjing University of Information Science \& Technology, China \\ * corresponding author
}

\begin{abstract}
Large errors of low-cost MEMS inertial measurement unit (MIMU) lead to huge navigation errors, even wrong navigation information. An integrated navigation system for unmanned vessel is proposed. It consists of a low-cost MIMU and Doppler velocity sonar (DVS). This paper presents an integrated navigation method, to improve the performance of navigation system. The integrated navigation system is tested using simulation and semi-physical simulation experiments, whose results show that attitude, velocity and position accuracy has improved awfully, giving exactly accurate navigation results. By means of the combination of low-cost MIMU and DVS, the proposed system is able to overcome fast drift problems of the low cost IMU.
\end{abstract}

Keywords: Low cost; MEMS; Inertial navigation; Integrated navigation

\section{INTRODUCTION}

Unmanned vessel (UV) is an effective tool in ocean exploitation. The recent upsurge of interest in ocean exploitation necessitates the development of small and lightweight UVs[1]-[5]. Precision navigation is one of the key technologies and of great importance for UVs and UV-based ocean surveying and exploitation. This paper focuses on mini-sized unmanned vessels, which have a limited weight, size and cost, making low cost navigation system the best, and most of time, the only choice.

This paper presents a navigation system scheme for UVs. Low cost Micro-Electro-Mechanical Systems (MEMS) based inertial measurement unit (IMU) is used, to determine the position, velocity and attitude of the UV, in which the IMU is fixed - this is so called inertial navigation system (INS). The operation of inertial navigation systems depends upon the laws of classical mechanics as formulated by Isaac Newton.
It is the process whereby the measurements provided by gyroscopes and accelerometers are used to determine the position, velocity and attitude of the vehicle in which they are installed. Inertial navigation system is entirely self-contained within the vehicle, in the sense that they are not dependent on the transmission of signals from the vehicle or reception from an external source. So it is widely used. But inaccuracies arise because of initial alignment errors, imperfections in the performance of the inertial instruments and limitations in the computational process. For high-precision IMU, the position error will increase slowly; so INS still give highprecision navigation information in not a long time. Yet for low cost MIMU, large errors (as shown in Table I) lead to huge navigation errors, even wrong navigation information even in a minute. It cannot be used in isolation. So we combined INS and doppler velocity sonar (DVS) to an integrated navigation system, to improve the accuracy of the inertial navigation system[6]-[9]. 
Tab. I. Functional specifications of MIMU used in this work

\begin{tabular}{|c|c|c|}
\hline Parameter & Gyroscope & Accelerometer \\
\hline Range & $\pm 150 \mathrm{deg} / \mathrm{s}$ & $\pm 10 \mathrm{~g}$ \\
\hline Bias & $0.1 \mathrm{deg} / \mathrm{s}$ & $0.5 \mathrm{mg} \times$ Range \\
\hline Bias stability & $0.02 \mathrm{deg} / \mathrm{s}$ & $0.1 \mathrm{mg} \times$ Range \\
\hline Scale Factor non-linearity & $0.5 \%$ & $0.5 \%$ \\
\hline
\end{tabular}

\section{THE INTEGRATED NAVIGATION SYSTEM DESIGN}

\section{INERTIAL NAVIGATION SYSTEM ERRORS EQUATION}

The navigational function is fulfilled by combining the measurements of vehicle rotation and specific force with knowledge of the gravitational field to compute estimates of attitude, velocity and position with respect to a predefined reference frame. A schematic representation of such an inertial navigation system is shown in Figure 1.

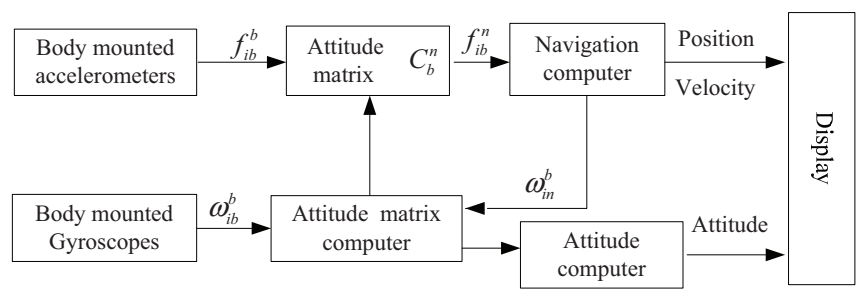

Fig. 1. Inertial navigation system schematic

\section{(1) Inertial navigation system errors equation}

Inertial navigation system attitude errors, velocity errors and position errors are as follows[10][11]:

$$
\left\{\begin{array}{l}
\dot{\varphi}^{n}=\delta \omega_{i e}^{n}+\delta \omega_{e n}^{n}-\left(\omega_{i e}^{n}+\omega_{e n}^{n}\right) \times \varphi^{n}+\varepsilon^{p} \\
\delta \dot{V}^{n}=\mathrm{f}^{n} \times \varphi^{n}+\nabla^{p}-\left(2 \delta \omega_{i e}+\delta \omega_{e n}\right) \times \dot{V}^{n}-\left(2 \omega_{i e}+\omega_{e n}\right) \times \delta V^{n} \\
\delta \dot{L}=\frac{\delta V_{y}}{R_{n}} \\
\delta \dot{\lambda}=\frac{\delta V_{x}}{R_{E}} \sec L+\frac{V_{x}}{R_{E}} \sec \operatorname{Ltg} L \delta L
\end{array}\right.
$$

Equ. (1) is inertial navigation system errors equation. It may be expressed as a matrix error equation as follows:

$$
\dot{X}_{I}=F_{I} X_{I}+W_{I}
$$

where

$$
X_{I}=\left[\begin{array}{lllllll}
\delta V_{E} & \delta V_{N} & \varphi_{E} & \varphi_{N} & \varphi_{U} & \delta L & \delta \lambda
\end{array}\right]
$$

\section{(2) Inertial sensor errors}

The inertial sensor errors are considered to be the forms of random bias and white noise as follows:

$$
\left\{\begin{array}{l}
\varepsilon=\varepsilon_{b}+w_{g} \\
\nabla=\nabla_{b}+w_{a}
\end{array}\right.
$$

where

$\varepsilon_{b}$ and $w_{g}, \nabla_{b}$ and $w_{a}$ are gyroscopes random bias and white noise, accelerometers random bias and white noise respectively, and:

$$
\left\{\begin{array}{l}
\dot{\varepsilon}_{b}=0 \\
\dot{\nabla}_{b}=0
\end{array}\right.
$$

Three gyroscopes random biases and three accelerometers random biases included, the system equation is as follows:

$$
\dot{X}=F X+W
$$

where

$X=\left[\begin{array}{llllllllllll}\delta V_{E} & \delta V_{N} & \varphi_{E} & \varphi_{N} & \varphi_{U} & \delta L & \delta \lambda & \nabla_{b x} & \nabla_{b y} & \varepsilon_{b x} & \varepsilon_{b y} & \varepsilon_{b z}\end{array}\right] ;$

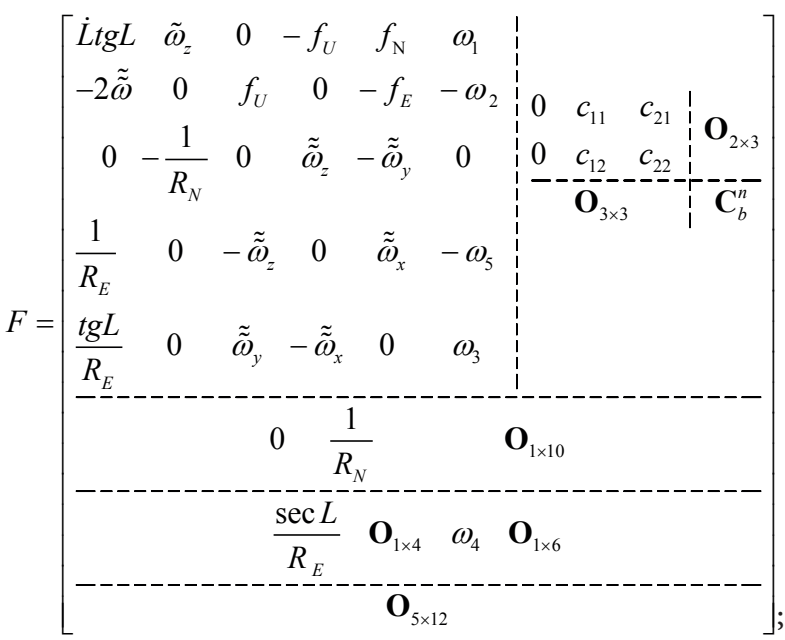

$W=\left[\begin{array}{llllllllllll}w_{v_{E}} & w_{v_{N}} & w_{\varphi_{E}} & w_{\varphi_{N}} & w_{\varphi_{U}} & w_{\delta L} & w_{\delta \lambda} & 0 & 0 & 0 & 0 & 0\end{array}\right]^{T} ;$

$c_{i j}$ - the element of direction cosine matrix $C_{n}^{b}$;

$\varepsilon_{b x}, \varepsilon_{b y}, \varepsilon_{b z}, \nabla_{b x}, \nabla_{b y}, \nabla_{b z}$ : gyroscopes random biases and accelerometers random biases respectively in $\mathrm{xb}, \mathrm{yb}, \mathrm{zb}$ axis of the body-fixed coordinate frame;

$\omega_{1}=\left(2 \omega_{i e} \cos L+\frac{V_{E}}{R_{E}} \sec ^{2} L\right) V_{N}, \omega_{2}=\left(2 \omega_{i e} \cos L+\frac{V_{E}}{R_{E}} \sec ^{2} L\right) V_{E}$

$\omega_{3}=\omega_{i e} \cos L+\frac{V_{E}}{R_{E}} \sec ^{2} L, \omega_{4}=\frac{V_{E}}{R_{E}} \sec ^{2} L \sin L, \omega_{5}=\omega_{i e} \sin L$

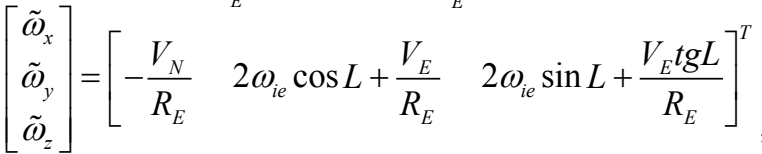

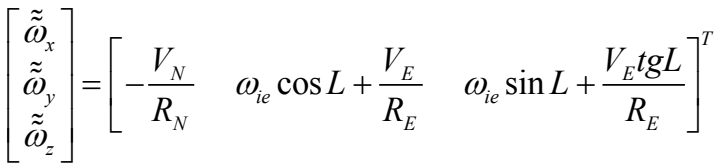




\section{THE INTEGRATED NAVIGATION SYSTEM DESIGN}

The integrated navigation system scheme is shown in Figure 2. INS and DVS are combined to a Kalman filter, to estimate and correct IMU biases and inertial navigation system errors, in order to overcome fast drift problems of the low-cost INS, then improve navigation accuracy[11] [12].

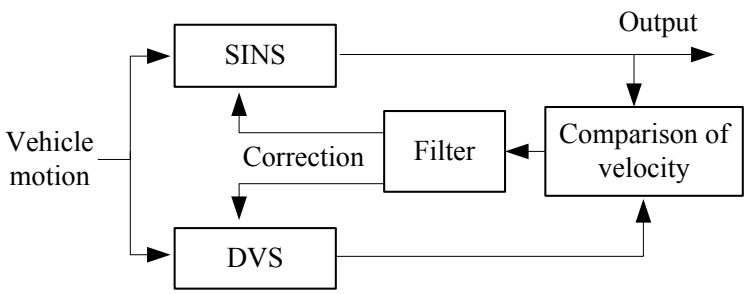

Fig. 2 Integrated navigation system schematic for unmanned vessel

\section{INTEGRATED NAVIGATION SYSTEM FILTER DESIGN}

\section{System equation}

Equation (5) is the Kalman filter system equation.

\section{Measurement equation}

The inertial navigation system output velocities are compared with DVS velocity measurements. The velocities difference of INS/DVS are used as Kalman filter measurements, to estimate and correct INS errors.

The inertial navigation system output velocities and DVS velocity measurements are as Equ. (6) and )7) respectively:

$$
\begin{gathered}
\left\{\begin{array}{l}
v_{I E}=v_{E}+\delta v_{E} \\
v_{I N}=v_{N}+\delta v_{N}
\end{array}\right. \\
\left\{\begin{array}{l}
v_{D E}=v_{E}+\delta v_{D E} \\
v_{D N}=v_{N}+\delta v_{D N}
\end{array}\right.
\end{gathered}
$$

where $\delta v_{D E}, \delta v_{D N}$ represent the errors in the DVS velocity measurements.

The differences between the DVS measurements and INS measurements are referred to as the filter measurements and are as follows:

$$
\begin{aligned}
\boldsymbol{Z}_{v} & =\left[\begin{array}{l}
v_{I E}-v_{D E} \\
v_{I N}-v_{D N}
\end{array}\right]=\left[\begin{array}{l}
\delta v_{E}-\delta v_{D E}+w_{1} \\
\delta v_{N}-\delta v_{G N}+w_{2}
\end{array}\right] \\
& =\left[\begin{array}{lll}
1 & 0 & \boldsymbol{O}_{2 \times 10} \\
0 & 1
\end{array}\right]_{2 \times 12}\left[\begin{array}{l}
\boldsymbol{X}_{\boldsymbol{I}} \\
\boldsymbol{X}_{D}
\end{array}\right]+\left[\begin{array}{l}
w_{1} \\
w_{2}
\end{array}\right] \\
& \triangleq \boldsymbol{H}_{v}\left[\begin{array}{l}
\boldsymbol{X}_{\boldsymbol{I}} \\
\boldsymbol{X}_{D}
\end{array}\right]+\boldsymbol{V}_{v}
\end{aligned}
$$

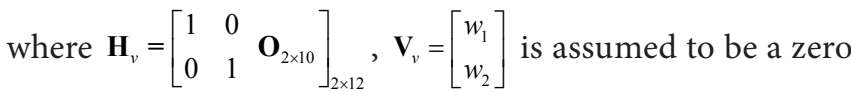
mean, Gaussian white-noise process.

In order to allow a discrete Kalman filter to be constructed, it is necessary to express the system error equation (5), the measurement equation (8) in discrete form as follows:

$$
\begin{gathered}
X_{k}=\varphi_{k, k-1} X_{k-1}+\Gamma_{k-1} W_{k-1} \\
Z_{k}=H_{k} X_{k}+V_{k}
\end{gathered}
$$

\section{Kalman filter process}

Equations (9) and (10) are the system and measurement equations needed to construct a Kalman filter. The equations for the Kalman filter take the following form for the integrated navigation system for unmanned vessel considered here.

\section{1) Filter prediction step}

The covariance matrix is predicted forward in time using the expression:

$$
P_{k / k-1}=\varphi_{k, k-1} P_{k-1} \varphi_{k, k-1}^{T}+\Gamma_{k-1} Q_{k-1} \Gamma_{k-1}^{T}
$$

and the state prediction equation:

$$
X_{k / k-1}=\varphi_{k, k-1} \hat{X}_{k-1}
$$

\section{2) Filter update}

The estimates of the errors in the inertial navigation system states are derived using:

$$
\hat{X}_{k}=X_{k / k-1}+K_{k}\left(Z_{k}-H_{k} X_{k / k-1}\right)
$$

and the covariance matrix is updated according to:

$$
P_{k}=\left(I-K_{k} H_{k}\right) P_{k / k-1}
$$

where

$$
K_{k}=P_{k / k-1} H_{k}{ }^{T}\left(H_{k} P_{k / k-1} H_{k}{ }^{T}+R_{k}\right)^{-1}
$$

\section{RESULTS}

\section{SIMULATION RESULTS}

According to sample data from the MIMU we used, the following errors sources are included.

Gyroscope white noise $=40 \% \mathrm{~h}$, gyroscope bias $=360 \% \mathrm{~h}$, accelerometer bias $=2 \mathrm{mg}$, accelerometer white noise $=2 \mathrm{mg}$.

Body motion: constant velocity $=15 \mathrm{~m} / \mathrm{s}$, initial latitude $=32^{\circ}$, longitude $=118^{\circ}$. Sine angular motion: initial yaw $=45 \mathrm{deg}$, 
pitch=9deg, roll=12deg, and frequency: $y a w=1 / 6$, pitch $=1 / 8$, roll $=1 / 10$.

The simulated INS errors are shown in Figure 3.
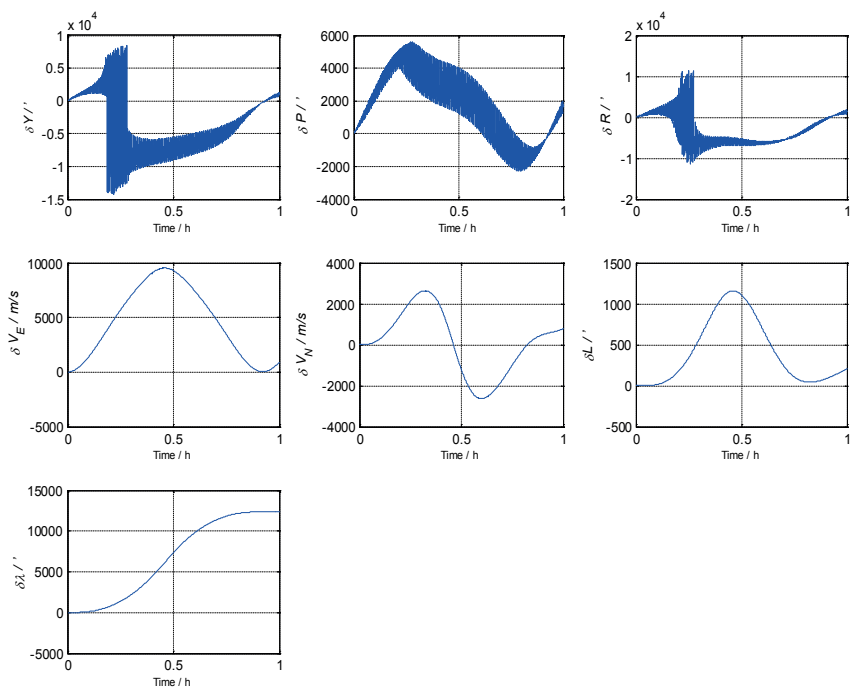

Fig. 3. Simulated INS errors

$\delta \mathrm{Y}, \delta \mathrm{P}, \delta \mathrm{R}, \delta \mathrm{VE}, \delta \mathrm{VN}, \delta \mathrm{L}$ and $\delta \lambda$ are yaw error, pitch error, roll error, east velocity error, north velocity error, latitude error, longitude error respectively.

Figure 3 shows huge navigation errors; this caused by the large errors from gyroscopes and accelerometers. The INS navigation results are worthless.

So we combined INS and DVS to the above integrated navigation system, then the simulated navigational accuracy is shown in Figure 4.
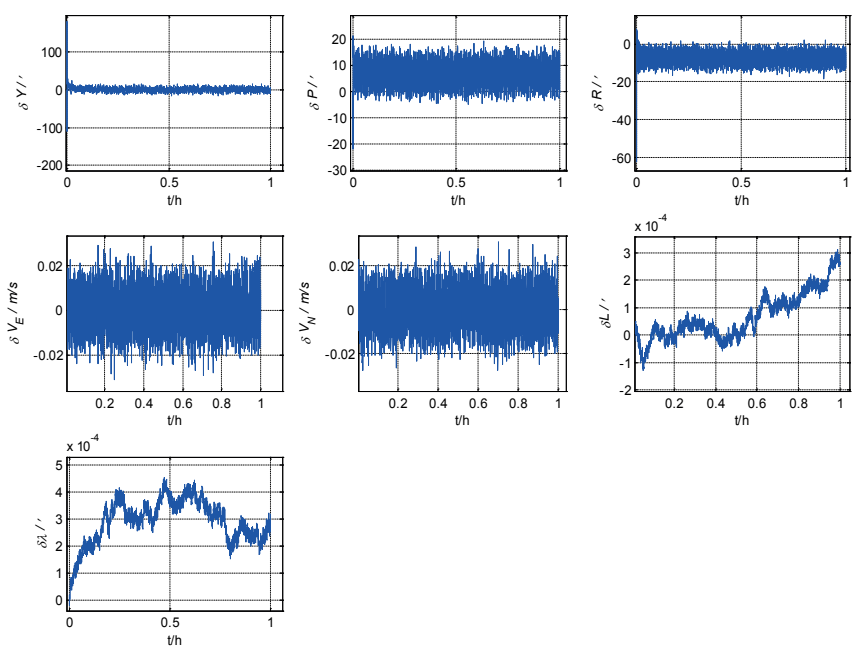

Fig. 4 Integrated navigation system errors

DVS velocity noise is $0.01 \mathrm{~m} / \mathrm{s}$. Figure 4 shows that the integrated navigation system improves navigational accuracy awfully, giving exactly accurate navigation results.

\section{SEMI-PHYSICAL SIMULATION RESULTS}

For further testing the integrated navigation system, semi-physical simulation was carried out. A low cost MIMU was used in this test. The MIMU was placed on a table under static coditions. About 5-10 minutes data are collected. The sample data are shown in Figure 5.
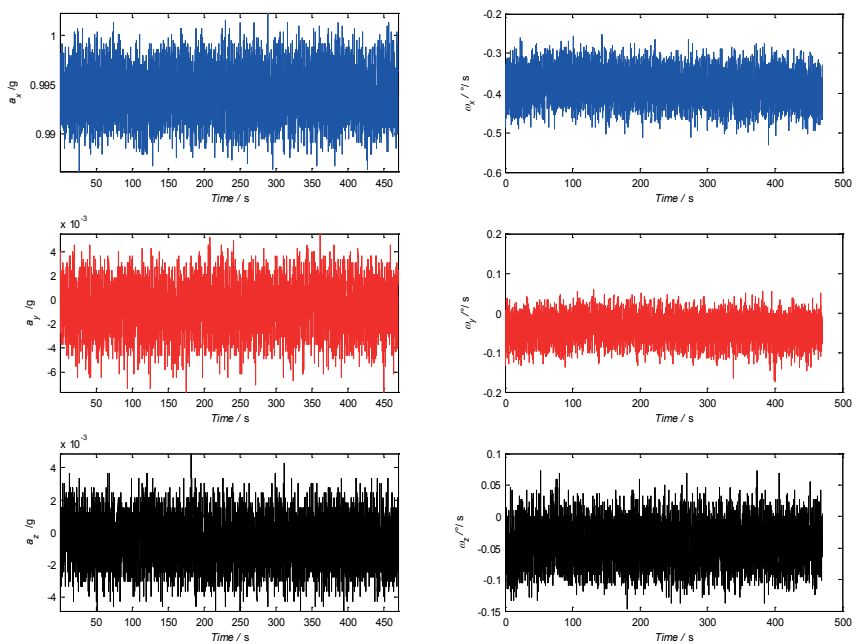

Fig. 5 MIMU Sample data under static conditions

ax, ay, az, $\omega_{x}, \omega_{y}, \omega_{z}$ are $\mathrm{x}$-axis, $\mathrm{y}$-axis and $\mathrm{z}$-axis specific forces and angular rates respectively.

Figure 5 shows that the gyroscopes and accelerometers have notable null bias errors.

Using these data, we have gotten INS results shown in Figure 6. The navigational errors are quite notable. So it's not worth a straw if working by itself. Then we have gotten INS results after IMU errors compensation, shown in Figure 7.
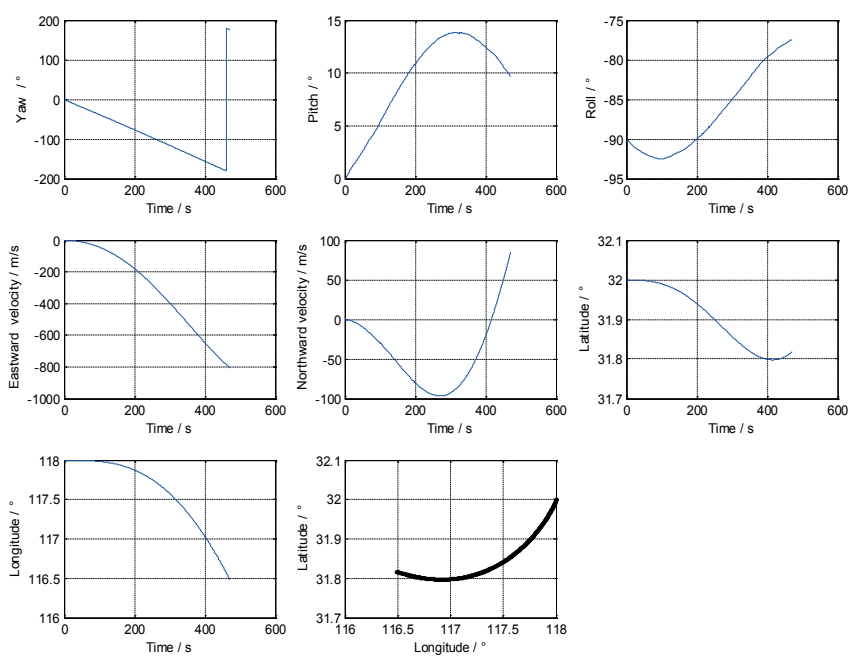

Fig. 6 INS navigational results - no IMU errors compensation 

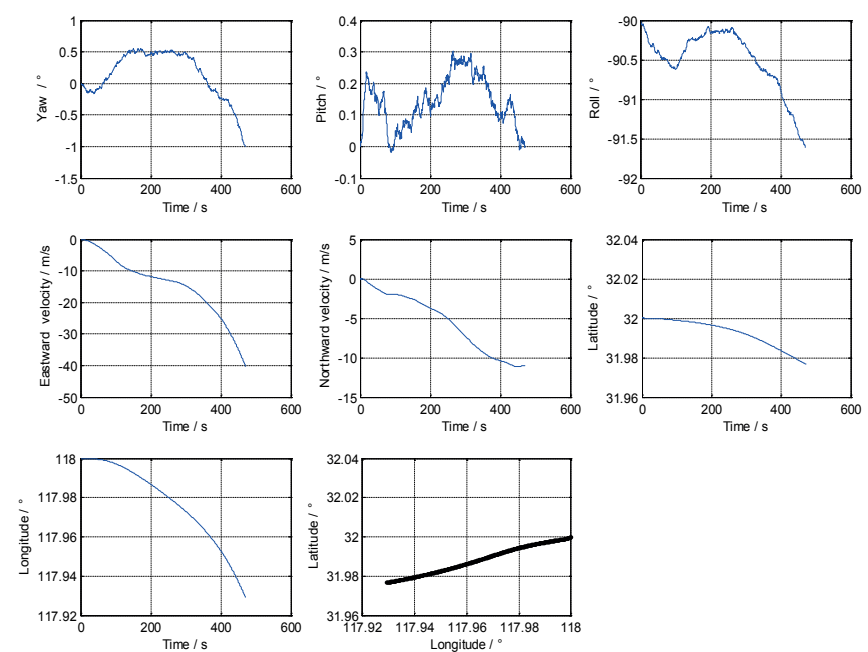

Fig. 7. INS results - after IMU errors compensation

The navigational accuracy is improved greatly. Yet there are still large errors, esp. for velocity and position.

The MIMU sample data are used to the above integrated navigation system, then the navigational results are shown in Figure 8 and Figure 9.
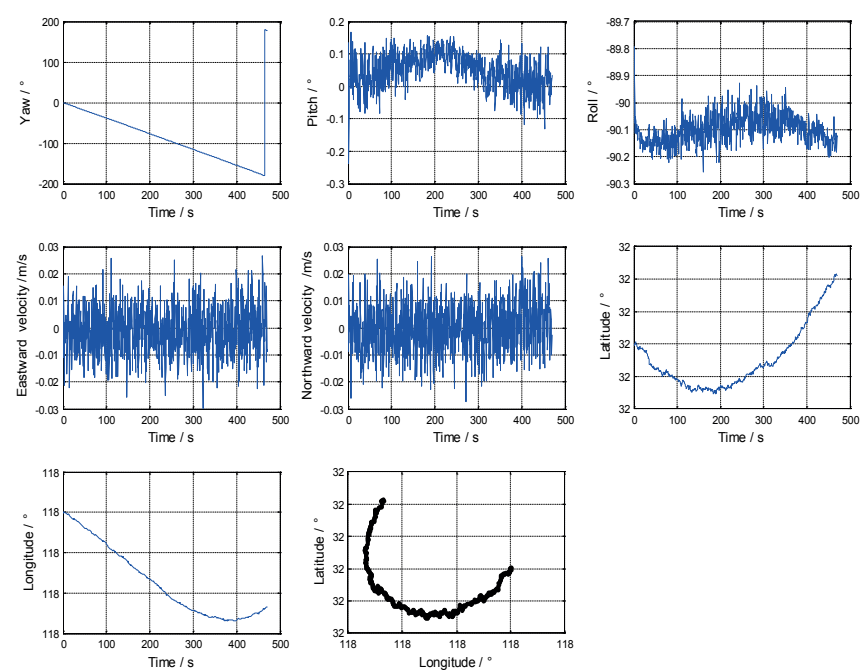

Fig. 8. Integrated navigation results - no IMU errors compensation.

Figure 9 shows that the results are much accurate. Yaw error is less than $0.3 \mathrm{deg}$; pitch and roll errors are less than $0.2 \mathrm{deg}$; by Matlab data statistics tools, we can see the maximum position error is $0.65 \mathrm{~m}$. The integrated navigation system designed above improved navigational accuracy awfully, giving exactly accurate navigation results.
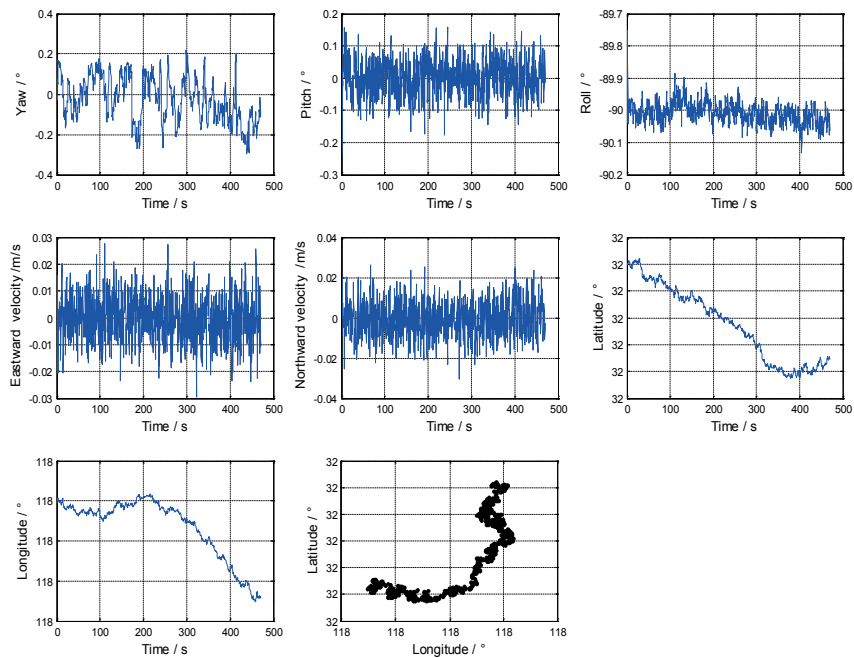

Fig. 9 Integrated navigation results - after IMU errors compensation

\section{CONCLUSIONS}

In this work, an unmanned vessel navigation system based on a low cost MEMS inertial measurement unit and a doppler velocity sonar has been presented. By fusing measurements from both sensor sources with a Kalman filter model, the problem that large errors of low cost MIMU will lead to huge navigation errors has been overcome. Simulation and semi-physical simulation results have demonstrated that the accuracy of the inertial navigation system has improved greatly.

\section{ACKNOWLEDGEMENTS}

This work was supported by project NSFC ( No. 61302189) and NSFJ (No. BK20160955).

\section{REFERENCES}

1. J. M. Daly, M. J. Tribou and S. L. Waslander, 2012. A nonlinear path following controller for an underactuated unmanned surface vessel. In: 2012 IEEE/RSJ International Conference on Intelligent Robots and Systems. Vilamoura. pp. 82-87.

2. M. H. Ghani, L. R. Hole, Ilker Fer, etal., 2014. The SailBuoy remotely-controlled unmanned vessel: Measurements of near surface temperature, salinity and oxygen concentration in the Northern Gulf of Mexico. Methods in Oceanography, (10), 104-121.

3. P. W. Pritchett, 2015. Ghost Vessels: Why the Law Should Embrace Unmanned Vessel Technology. Tulane Maritime Law Journal, 40(1), 197.

4. Y. Man, M. Lundh, T. Porathe, etal., 2015. From desk to field - Human factor issues in remote monitoring and 
controlling of autonomous unmanned vessels. Procedia Manufacturing, (3), 2674-2681.

5. J. M. Larrazabal, M. S. Peñas, 2016. Intelligent rudder control of an unmanned surface vessel. Expert Systems with Applications, (55), 106-117.

6. L. Zhang, Z. Xiong, J. Lai, etal., 2016. Optical flowaided navigation for UAV: A novel information fusion of integrated MEMS navigation system. Optik, (127), 447-451.

7. M. Morgado, P. Oliveira, and C. Silvestre, 2010. Design and experimental evaluation of an integrated USBL/INS system for AUVs. In: IEEE International Conference on Robotics and Automation, Alaska, pp.4264-4269.

8. Y. Geng, R. Martins, J. Sousa, 2010. Accuracy Analysis of DVL/IMU/Magnetometer Integrated Navigation System using Different IMUs in AUV. In: IEEE International Conference on Control and Automation, Xiamen, pp. 516-521.

9. C. Eling, L. Klingbeil and H. Kuhlmann, 2015. RealTime Single-Frequency GPS/MEMS-IMU Attitude Determination of Lightweight UAVs. Sensors, (15): 26212-26235.

10. Y. Qin, 2006. Inertial navigation. Science Press, Beijing, 355-361.

11. M. S. Grewal, A. P. Andrews, and C. G. Bartone, 2013. Global navigation satellite systems, inertial navigation, and integration, Third edition. John Wiley \& Sons, Inc., Hoboken, New Jersey.

12. V. Awale, H. B. Hablani, 2015. Fusion of Redundant Aided-inertial Sensors with Decentralised Kalman Filter for Autonomous Underwater Vehicle Navigation. Defence Science Journal, 65(6), 425-430.

\section{CONTACT WITH THE AUTHOR}

\section{Changsong Yang}

School of Information \& Control

Nanjing University of Information Science \& Technology

Nanjing, 210044

China 